\title{
IV. Handeln unter Bedingungen des globalen Wandels
}

Sonja Germer, Karl-Dieter Keim, Matthias Naumann, Oliver Bens, Rolf Emmermann, Reinhard F. Hüttl

Die diversen Auswirkungen des globalen Wandels erfordern in den betroffenen Regionen spezifische, den jeweiligen Bedingungen angepasste Handlungsstrategien und Maßnahmen. Hierfür erste Vorschläge zu entwickeln war ein Ziel der interdisziplinären Arbeitsgruppe Globaler Wandel - Regionale Entwicklung. Im Folgenden sollen daher zunächst übergeordnete Herausforderungen des globalen Wandels skizziert werden, die alle untersuchten Themenfelder betreffen. Daran schließt die Darstellung von Brückenprinzipien an, die eine Handlungsorientierung für den Umgang mit den Folgen des globalen Wandels bieten können. Am Ende steht ein Ausblick auf weitergehende Forschungsarbeiten.

\section{Übergeordnete Herausforderungen des globalen Wandels ${ }^{1}$}

Zur Bewältigung der Folgen des Umweltwandels für den Landschaftswasserhaushalt (Kapitel I), der Auswirkungen veränderter Landnutzungen für Wasserressourcen (Kapitel II) wie auch des Wandels von Denk- und Handlungsmustern bei der Gestaltung von Infrastruktur (Kapitel III) lassen sich allgemeine Herausforderungen identifizieren, die alle vorgenannten Themenfelder gleichermaßen betreffen. Dazu zählten erstens die Bedeutung unterschiedlicher räumlicher und zeitlicher Maßstabsebenen, zweitens eine wachsende Unsicherheit und Flexibilisierung von Entscheidungskontexten, drittens das Handeln unter Bedingungen des NichtWissen und viertens die Entstehung von neuen Akteurskonstellationen.

Diese Herausforderungen stellen keine analytisch trennscharfen Kategorien dar, sondern stehen für Problemkomplexe des globalen Wandels, die einander überlappen, verstärken und teilweise in einem Spannungsverhältnis zueinander stehen. Gemeinsam ist den verschiedenen Herausforderungen, dass sie bestehende Instrumente und Verfahren der Problembearbeitung infrage stellen. Um allgemein gültige Handlungsorientierungen für regionalspezifische Antworten auf den globalen Wandel zu entwickeln, ist es daher notwendig, diese übergreifenden Herausforderungen zu präzisieren.

\footnotetext{
${ }^{1}$ Dieser Abschnitt fasst zentrale Ergebnisse einer Diskussion aller Mitglieder im Rahmen der Plenumssitzung der IAG Globaler Wandel - Regionale Entwicklung zusammen.
} 


\section{Bedeutung unterschiedlicher räumlicher und zeitlicher Maßstabsebenen}

Die unterschiedlichen räumlichen und zeitlichen Maßstabsebenen, deren Bedeutungswandel und Interaktion, bilden eine zentrale Herausforderung für Entscheidungsträger im Rahmen aller hier betrachteten Bereiche des globalen Wandels. Denn sowohl die Veränderungen des globalen Wandels als auch die Reaktionen darauf verlaufen nicht einheitlich in Raum und Zeit (u. a. Rosswall et al. 1988). Dies betrifft etwa die Emission von Treibhausgasen, die global-langzeitig wirken, deren konkrete Wirkungen sich jedoch lokal-kurzzeitig ereignen können. Auch Prozesse, die in Modellen abgebildet werden, können sich je nach Skala unterschiedlich verhalten und sind zum Beispiel linear, nicht-linear oder schwellenwertabhängig (Ehleringer et al. 1993). Auch sind Klimaprojektionen nicht unbedingt von einer räumlichen Skala auf eine andere übertragbar (u. a. Zimmermann 2007; Walkenhorst \& Stock 2009).

Bei der Landnutzung und den Agrarmärkten sollte ebenfalls die jeweilige räumliche und zeitliche Maßstabsebene beachtet werden. So haben etwa die rechtlichen Regelungen für Biogasanlagen eine zeitliche wie auch räumliche Dimension. Die aktuellen Förderungen von Biogasanlagen haben Auswirkungen für die Pflanzenproduktion und somit auch für die Landnutzung, die weit über den zeitlichen und räumlichen Rahmen der Fördermaßnahme hinausgehen. Ein weiteres Beispiel betrifft die Vorratshaltung von Getreide und damit die räumliche und zeitliche Ebene der Betrachtung von Agrarmärkten. So bestehen 2010/2011 weltweit im Vergleich zu den vorangegangenen drei Jahren die geringsten Vorräte an Getreide (IGC 2011), so dass Staaten dazu übergehen, sich über Bodenerwerb im Ausland langfristig ihre Versorgung mit landwirtschaftlichen Produkten zu sichern (Fritz 2009). Der räumliche wie zeitliche Fokus der Sicherung der Nahrungsmittelversorgung erweitert sich damit. Es ist daher zu fragen, was globale Veränderungen in der Landwirtschaft für einzelne Betriebe bedeuten werden (Hagedorn, S. 86-92).

Politische Eingriffe und Gestaltung können zum Teil nicht wirksam werden, wie der Klimawandel oder die Finanzkrise eindrücklich zeigen, da die bisherigen räumlichen Bezugseinheiten, wie etwa der Nationalstaat oder die Europäische Union, zu eng gefasst sind hinsichtlich des globalen Ausmaßes der Veränderungen und deren Ursachen. In diesem Zusammenhang beziehen sich politische Maßnahmen auf andere räumliche Ebenen als die, auf denen grundlegende Entscheidungen der Finanzoder Klimapolitik getroffen werden. Die Frage räumlicher Maßstabsebenen wird in der Humangeographie von Swyngedouw und anderen mit Begriffen des „Rescaling", d.h. der Dynamisierung des Handelns quer zu verschiedenen räumlichen Maßstabsebenen, theoretisch konzeptionalisiert (Swyngedouw 1997, 2010; Wissen et al. 2008). Aber auch für zeitliche Maßstabsebenen gilt, dass sich politische Maßnahmen auf unterschiedliche Zeiträume auswirken. So ist zu bestimmen, auf welche zeitlichen Horizonte sich Entscheidungen beziehen und für wie viele Generationen Verantwortung übernommen werden kann. 


\section{Unsicherheit und Flexibilisierung}

Unsicherheit und Flexibilisierung bilden zentrale Rahmenbedingungen für regionale Strategien im Umgang mit dem globalen Wandel. Unsicherheit meint hier die Ungewissheit über die zukünftige Entwicklung des globalen Wandels und seiner regionalen Ausprägungen aufgrund von unvollkommener Information sowie unzureichender Kenntnis der zugrunde liegenden Prozesse.

Grundsätzlich sind diejenigen Systeme und Akteure überlegen, die besser mit Unsicherheiten umzugehen wissen. Auch wenn Unsicherheiten im Zuge des globalen Wandels wachsen, ist der Umgang mit diesen erlernbar (Renn et al. 2007). Beispielsweise kann ein besseres Verständnis der Aussagekraft von Mittelwerten und Spannbreiten von Klimawerten oder auch von Szenarien vermittelt werden. Beispiele sind die in der Klimaforschung bereits etablierten Multi-Modell-Ansätze, bei denen die entsprechenden Parameter mit verschiedenen verfügbaren Modellen berechnet und anschließend verglichen werden (Meehl et al. 2007). Weiterhin gilt, dass nicht alle Unsicherheiten zwangsläufig zu Verunsicherungen führen. Die Referenzhorizonte für Unsicherheiten sind sehr unterschiedlich und verändern sich im historischen Verlauf. Da Beobachtungen verdichtet und Modellierungen beständig verbessert werden, ändert sich das Wissen stetig. In diesem Zusammenhang kann die Informationstechnologie Instrumente zur Verfügung stellen, um die Erzeugung und Verbreitung von neuem Wissen zu beschleunigen bzw. Wissen schneller zu aktualisieren (Köstner/Kuhnert, S. 43-49).

Mit wachsender Unsicherheit wächst auch die Notwendigkeit zur Flexibilität bei der Planung und Findung von Entscheidungen. Beispielsweise sollten Projektplanungen auf eine Zeitachse gelegt und in Stufen unterteilt werden, um eine spätere Anpassung an bislang noch nicht bekannte Anforderungen zu ermöglichen. Gleichzeitig sollte das Projektmanagement von einem Risikomanagement begleitet werden, um sich verändernde Ausgangsbedingungen zu identifizieren (Schelle et al. 2008). Sinnvoll ist auch ein modulares Planen, bei dem der Planungsprozess in kleinere Einheiten unterteilt wird (Haaren et al. 2005). Dabei sollte zudem beachtet werden, dass die Gegenstände der Planung zusätzliche neue Funktionen bekommen können, wie Beispiele von vorhandenen Infrastrukturen wie Bahnhöfe oder Talsperren zeigen (Barlösius/Keim/Meran/Moss/Neu, S. 164 f.). Viele Planungen erfolgten bisher vor allem deduktiv, wobei ausgehend von einem Ziel verschiedene Maßnahmen auf dem Weg dorthin abgeleitet wurden. Demgegenüber geht das modulare Planen induktiv vor, womit eine stärkere Ergebnisoffenheit verbunden ist. Dabei ist die bisherige Grundannahme vieler Planungen hinsichtlich eines dauerhaften Wirtschafts- und Bevölkerungswachstums kritisch zu hinterfragen.

Flexibilität ist jedoch nicht nur im Rahmen von langfristigen Großprojekten gefragt, sondern auch in der jährlichen, das heißt vor allem operativen Planung der Landnutzung und Agrarproduktion, denn das Wetter, die künftige Nachfrage, die EU-Förderung etc. sind grundsätzlich mit Unsicherheiten verbunden. Daher sollte einerseits der Landwirt beim Spektrum von Produkten und bei den Anbauformen flexibel sein. Für die Pflanzen- und Tierzüchtung werden andererseits Stresstoleranz und physiologische Flexibilität als Züchtungsziele immer wichtiger (Broer) 
Brunsch, S. 129-134). Hierbei ist zu beachten, dass Stresstoleranz häufig mit Produktivitätseinbußen einhergeht, was wiederum einen höheren Wasserbedarf zur Produktion der gleichen Mengen Biomasse bzw. Erntegut bedeutet.

\section{Handeln unter Bedingungen des Nichtwissens}

Alle Entscheidungen sind mit der Herausforderung konfrontiert, dass Handeln häufig unter Bedingungen des Nichtwissens stattfindet. Auch wenn Wissen über die Trends der künftigen Entwicklung vorhanden ist, sollten weitere Optionen offengehalten werden. Im Sinne einer „Governance of Preparedness“ (Medd \& Marvin 2005) sind auch unsichere und bislang nicht bekannte Entwicklungen zu berücksichtigen. Für den Fall, dass Entwicklungen falsch eingeschätzt oder ungünstige Entscheidungen in der Vergangenheit getroffen wurden, sollte eine schnelle Umorientierung möglich sein.

Da Entscheidungen häufig unter Bedingungen des Nichtwissens getroffen werden, ist eine Orientierung am „No-Regret“-Prinzip sinnvoll: Maßnahmen sollten stets die Robustheit bzw. die Resilienz eines Gesamtsystems fördern (Hallegatte 2009). Die Bodenverbesserung mit der Verbesserung des Humus- und Wasserhaushalts kann so für den Bereich Landwirtschaft als „No-Regret“-Maßnahme angesehen werden, da sie dazu beiträgt, Schwankungen im System abzupuffern, aber auch einen positiven Effekt auf die landwirtschaftliche Produktion haben kann, wenn die erwarteten Schwankungen nicht eintreten. Die Komplexität von Systemen kann die Stärkung der Robustheit erschweren. Also sollten die Akteure den systemischen Zusammenhang überblicken, in seiner Besonderheit erkennen und hinsichtlich seiner Ausgestaltung kommunizieren und handhaben können. Zur Formulierung von „No-Regret"-Maßnahmen bedarf es wie bei der modularen Planung einer Kombination von induktiven und deduktiven Ansätzen. Dies bedeutet, dass der Weg zum Ziel optimiert wird, aber das Ziel auch mehrfach neu definiert werden kann. Die „No-Regret“-Strategie stellt dabei eine grundsätzliche Abkehr von bisherigen angebotsorientierten und investiven Planungen, etwa im Infrastrukturbereich dar, die in der Vergangenheit Systeme mit mangelnder Beweglichkeit und hoher Pfadabhängigkeit schufen (Barlösius/Keim/Meran/Moss/Neu, S. 149 f.).

Ein weiterer Punkt betrifft die Tatsache, dass es kein endgültig sicheres Wissen gibt, aber abgesichertes Wissen geben kann. Es sind Kriterien und Verfahren zu identifizieren, die Aussagen darüber erlauben, wann Wissen in Bezug auf ein bestimmtes Problem ausreichend abgesichert ist, um eine Entscheidung treffen zu können. Daher sollte beim Handeln unter Bedingungen des Nichtwissens angestrebt werden, eine Lösung auszuhandeln, zu der alle Beteiligten stehen können, obwohl sie wissen, dass sie nur einen Teil der für die Entscheidung notwendigen Informationen sicher kennen. Die Befähigung zur Kommunikation wäre dann im Zuge des Aushandlungsprozesses Garant für den geeigneten Umgang mit Folgen des globalen Wandels im Sinne eines kommunikativ-konstruktiven Vorausdenkens bzw. eines kommunikativ-praktischen Handelns, wenn Veränderungen eingetreten sind (pers. Mitteilung H.-E. Tenorth, 2010). 


\section{Entstehen neuer Akteurskonstellationen}

Neue Akteurskonstellationen sind als eine Erweiterung der bisher beteiligten Stakeholder und als eine Veränderung der Rollen bzw. Rollenverständnisse der beteiligten Akteure zu verstehen. Da die neuen Problemlagen wie Klimawandel, veränderter Landschaftswasserhaushalt und demographischer Wandel komplex sind und kommunale Grenzen überschreiten, sind Lösungsvorschläge immer weniger von einem Ressort oder einer Wissenschaftsdisziplin allein zu erwarten. So führt die wachsende Zusammenarbeit von Wissenschaft, Fachbehörden und Landnutzern zur Erweiterung der beteiligten Stakeholder. Diese Kooperationen werden im Zuge von Querschnittsaufgaben wie der Gestaltung neuer Anpassungsmaßnahmen als Reaktion auf den Klimawandel bzw. Veränderungen des Landschaftswasserhaushaltes oder den demographischen Wandel enger und eröffnen die Möglichkeit, integrierende Maßnahmen für die Ressourcenbewirtschaftung oder auch die Infrastrukturplanung zu entwickeln. Erste strukturelle Ansätze sind, dass z.B. Referate zu Klimaschutz und Klimawandel nicht in den Fachabteilungen, sondern unter Grundsatzangelegenheiten geführt werden, wie dies bei Klimareferaten in der Landesverwaltung des Freistaats Sachsen der Fall ist. Wichtig ist, dass zentrale Ansprechpartner benannt sind. So entstehen neue Akteure, die in laufenden Projekten z.B. als Klimaanpassungsbeauftragte (Verwaltung) oder -manager (Wirtschaft) bezeichnet werden (KLIMZUG-Nordhessen). Zudem sind erweiterte Akteurskonstellationen in der Landwirtschaft festzustellen. Landwirte sind gefordert, sich mit einer immer breiteren Palette von Technologien, Produkten und Märkten auseinanderzusetzen. Hierzu zählen beispielsweise Solar- oder Biogasanlagen oder auch die Nutzung von GPS-Systemen. Hinzu kommt, dass sich die wissenschaftlichen Bezugsdisziplinennicht nur für die Landwirtschaft - verändert haben. Landwirte benötigen daher nicht nur erweitere Netzwerke, sondern auch ein komplexeres Wissen, um effizient einen Betrieb führen zu können.

In diesem Kontext nehmen Akteure veränderte Rollen ein. Landwirte, die sich herkömmlich mit der Produktion von Futter- und Lebensmitteln beschäftigten, übernehmen bei (Teil-)Umstellung der Produktion auf nachwachsende Rohstoffe und Inbetriebnahme von dezentralen Biogasanlagen zunehmend auch die Rolle eines Energiewirtes (Plieninger et al. 2009; Plieninger et al. 2008). Bei langjährigem negativen Landschaftswasserhaushalt ist es bei entsprechendem finanziellen Anreiz auch denkbar, dass Land- und Forstwirte als „Wasserwirte“ tätig werden, indem sie ihre Landbewirtschaftungsmethoden bzw. Anbausysteme so ausrichten, dass sie einerseits wassersparend sind und andererseits die Grundwasserneubildung begünstigen (WWAP 2003).

Wo neue Akteurskonstellationen infolge von Querschnittsaufgaben entstehen, besteht Konfliktpotenzial. So können Akteure auf bestehende administrative Grenzen oder auf Grenzen zwischen bestehenden Ressorts stoßen, oder es treffen Akteure mit unterschiedlichen Denkweisen aufeinander. Außerdem bestehen eventuell noch ungenutzte Synergien. Die Ressorts sind hinsichtlich der Instrumentarien wie Ordnungsrecht oder Fördermittel sehr unterschiedlich ausgestattet und ausgerichtet. Im Hinblick darauf sollten bereits vorhandene oder zukünftig mögliche Synergien 
und Komplementaritäten zwischen bestehenden Ressorts näher untersucht und Inkompatibilitäten zwischen Ressorts aufgelöst werden.

Querschnittsaufgaben führen auch zu einem Bedarf an neuen Formen der Bürger- und Nutzerbeteiligung, die eine Ausweitung von bestehenden Instrumenten der Partizipation umfassen. Diese sind notwendig, um die Akzeptanz für verschiedene Projekte nicht nur im Infrastrukturbereich, sondern auch bei Landnutzungen etc. zu erhöhen. In vielen Themenfeldern erweitern Bürgerinitiativen die bisherigen Akteurskonstellationen, wie Konflikte um gentechnisch veränderte Pflanzen oder Infrastrukturprojekte zeigen (Joss \& Durant 1995). Dem Interesse von Bürgern und Nutzern an einer Beteiligung bei der Gestaltung künftiger Projekte sollte durch entsprechende Partizipationsmöglichkeiten Rechnung getragen werden. Als Beispiel hierfür stehen aktuelle transdisziplinäre Forschungsprojekte, die das Einbeziehen von Stakeholdern aus Verwaltung, Politik und dem Nutzerkreis an den Beginn der Projektplanung stellen (KirchnerHeßler et al. 2007). Bürger- und Nutzerbeteiligung ist jedoch nicht immer leicht zu realisieren, und eine Akzeptanz der Projekte ist trotz früher Bürgerbeteiligung nicht immer garantiert. Nicht alle Probleme sind durch eine Optimierung der Partizipation allein lösbar, und nicht alle Entscheidungen sind kompromissfähig oder können zur Disposition gestellt werden. Dennoch müssen Wege gefunden werden, die eine umfassende Beteiligung von Bürgern und Nutzern einerseits ermöglichen, die es andererseits aber erlauben, dass am Ende des Beteiligungsund Aushandlungsprozesses auch eine Entscheidung für einen Lösungsbeschluss getroffen werden kann (Rittel 1992). Da diese Fragen grundsätzlich die Zukunft der Demokratie betreffen, dürfen der Bürger und dessen Beteiligung nicht als „notwendiges Übel“ betrachtet, sondern sollten als gemeinsamer und gewinnbringender Prozess zur Gestaltung künftiger Planungen angesehen werden. Der Bürger darf dabei nicht primär als „Betroffener“, sondern sollte auch als Experte gesehen werden (Calheiros et al. 2000; Rittel 1992). Beispiele wie die von Bürgern initiierten energieautarken Gemeinden zeigen, dass die starre Dichotomie von „Entscheidern vs. Bürgern“ zunehmend obsolet wird, da Bürgerbeteiligung durchaus proaktiv und gestaltend wirken kann.

Allgemein gilt, dass sich Akteurskonstellationen während eines Planungsprozesses, der sich mitunter über mehrere Jahrzehnte hinzieht, verändern können und es zur Entstehung von unerwarteten Konstellationen kommen kann. Damit stellt sich auch hier die Notwendigkeit, Planungen so zu gestalten, dass spätere Veränderungen hinsichtlich der Nutzung bzw. Nutzergruppen noch berücksichtigt werden können. Die Akzeptanz von Investitionsvorhaben kann dadurch vergrößert werden, dass nicht alles ad hoc als Großprojekt entschieden und geplant wird. Wenn Verfahren flexibel gestaltet werden, sehen sich Bürger und Nutzer nicht im Nachhinein gezwungen, Möglichkeiten der Mitwirkung und die dazu erforderliche Transparenz der Vorgänge und Vorentscheidungen einzuklagen.

Schließlich sind auch Transparenz und ein stetiger Informationsfluss wesentliche Voraussetzungen, um neuen Akteurskonstellationen und Beteiligungsformen gerecht zu werden. Hierzu zählen beispielsweise neue Beratungs-, Informations- und Vermittlungsangebote zum Klimawandel und dessen Folgen für Landnutzung und 
Nutzung natürlicher Ressourcen in Bund, Ländern und Regionen, wie das Deutsche Klima-Konsortium (DKK), das Climate Service Center (CSC), das Kompetenzzentrum für Klimafolgen und Anpassung (KomPass) und regionale Klimabüros, wie etwa die Klimaplattform Brandenburg-Berlin. Wie integrierte regionale Ansätze von Anpassungen an den Klimawandel aussehen könnten, wird derzeit in den KLIMZUG-Projekten, für Brandenburg in INKA-BB, entwickelt (BMBF 2009). Eine wirkungsvolle Möglichkeit, mit Konflikten hinsichtlich neuer landwirtschaftlicher Produktionsmethoden umzugehen, ist es, Information über die konkreten Inhalte von neuen Projekten zugänglich und gleichzeitig sowohl die Chancen als auch das Nichtwissen transparent zu machen.

Die Bewältigung der hier aufgeführten übergeordneten Herausforderungen des globalen Wandels erfordert die Berücksichtigung neuer Rahmenbedingungen von Politik und Planung wie auch Handlungsprinzipien, die es erlauben, auch unter Ungewissheit Entscheidungen zu treffen und diese angesichts neuer Entwicklungen und bei Erkenntnisgewinn entsprechend anzupassen und weiterzuentwickeln. Hierfür können Brückenprinzipien wertvolle Anregungen liefern.

\section{Brückenprinzipien als Handlungsorientierung für den Umgang mit dem globalen Wandel}

Häufig ist zu beobachten, dass Handelnde umstandslos zur Formulierung von Lösungsvorschlägen schreiten, sobald die Herausforderungen identifiziert sind. Tatsächlich besteht jedoch zwischen der Definition der Situation und den zu treffenden Entscheidungen eine Begründungslücke, aus Ist-Aussagen lassen sich logisch keine Soll-Aussagen ableiten. Daher wird empfohlen, den normativen Zwischenschritt transparent zu machen, indem herangezogene Prinzipien, Präferenzen und Wertungen dargestellt werden.

Brückenprinzipien sind Hilfsmittel, die angewandt werden zur kritischen Bewertung von Lösungsvorschlägen und zur Formulierung von Handlungsoptionen. Sie kommen zum Einsatz, wenn außerwissenschaftliche (politische, gesellschaftliche) normative Entscheidungen samt ihrer Wertbezüge mithilfe theoretisch fundierter empirischer Ergebnisse kritisiert und in einem weiteren Schritt Vorschläge gemacht werden sollen, wie politische oder gesellschaftliche Probleme auf wissenschaftlicher Grundlage rational zu lösen sind (Keim 2009). Betrachtet man das prekäre Verhältnis zwischen Wissenschaften und Politik, so bieten Brückenprinzipien die Chance, die Kommunikation zwischen diesen beiden Teilsystemen in diesem Sinne zu verbessern.

Die Anwendung solcher Brückenprinzipien dient dazu, die Zahl der normativ aufgeladenen Problemlösungen deutlich zu reduzieren. Damit wird ein Weg geebnet für eine stärkere Rationalität bei der Regulierung von Gemeinschaftsgütern, die als Antwort auf die Folgen des globalen Wandels neu organisiert werden. Brückenprinzipien sollten folglich nicht mehr als genuin wissenschaftliches Mittel der Rationalitätssteigerung, sondern als Mittel der rationaleren Gestaltung der Kommunikation zwischen den entsprechenden Teilbereichen regionaler Steuerung verstanden werden, also der 
verschiedenen wissenschaftlichen Teildisziplinen, der Raum-, Infrastruktur-, Agrarund Ernährungspolitik sowie der dazugehörigen Wirtschafts- und Technikbereiche.

Die Brückenprinzipien werden hier in Form von sieben Postulaten präsentiert, die sich aus ihnen folgern lassen. Zusätzlich soll eine Übersicht konkreter Fragen die Anwendung im Einzelfall erleichtern (Tab. 10).

- Das wichtigste Brückenprinzip lautet: „Sollen impliziert Können“ oder, logisch äquivalent, „Nicht-Können impliziert Nicht-Sollen“. Was politisch geplant werden soll, lässt sich nicht betreiben, wenn es aufgrund des wissenschaftlichen Wissens nicht möglich ist. Daraus resultiert ein Realisierbarkeits-Postulat, das als kritisches Prüfkriterium an politische Entscheidungen im Hinblick auf den globalen Wandel angelegt werden sollte (Barlösius/Keim/Meran/Moss/Neu, S. 170 f.). Umgekehrt eröffnet der Stand des wissenschaftlichen Wissens einen Korridor an möglichen gesellschaftlichen bzw. politischen Lösungen.

- Als zweites Brückenprinzip kann formuliert werden: Ungewissheit und Chaos evozieren einen Hang zur Ordnung. Ordnung wird hier nicht rechtlich verstanden, sondern als fundamentales (anthropologisches) Wirklichkeitsvertrauen, als Heilen (von Unheil oder Katastrophenfolgen), das gesellschaftlich durch schützende Sinnstrukturen aufgenommen und über unterschiedliche gesellschaftliche Medien gestaltet wird. Das Ordnungs-Postulat nimmt in den Blick, inwieweit Problemlösungen einen messbaren Beitrag dazu leisten, Unheil bzw. Ungewissheit und Chaos zu zähmen, zu reduzieren, einzuhegen. Anders gewendet: Anomische Situationen (Situationen der Ordnungs- und Normlosigkeit) sollen durch Sinnstrukturen oder auch sozialtechnologische Systeme vermieden oder aber, sofern bereits vorhanden, spürbar abgebaut werden. Hingegen wären Lösungen zu kritisieren, die das $\mathrm{Ma}$ an Unheil/Ungewissheit erhöhen, ohne hinreichende Stabilisierungsvorschläge mitzuliefern.

- Das dritte Brückenprinzip besteht darin, auf die Urform des Spiels zurückzugreifen, ein kulturanthropologisches Grundelement: Gehe komplexe Lösungen spielerisch an. Spiele besitzen eigene Regeln, die auf Zeit gelten und die Regeln der „,normalen“ Welt außer Kraft setzen. Dadurch eröffnen sie befreiende, bereichernde Erfahrungen und überraschende Lösungen für spielerisch zu bearbeitende Probleme. Zum Spiel zählen auch Neugier- und Risikoverhalten sowie Mut und Spontaneität. Auf unser Aufgabenfeld angewandt, bedeutet ein solches Experimentier-Postulat, Spielräume für experimentelle Modelle und kreative Projekte zu schaffen. In die möglichen Handlungsoptionen sollen also situativ Erprobung und Improvisation eingebaut werden. Gerade für Situationen mit einem hohen Grad an Nichtwissen erscheint das Experimentieren unter definierten Randbedingungen eine angemessene Handlungsweise.

- Ergänzend ist ein Kontext-Postulat notwendig und hilfreich: Berücksichtige besondere Kontexte, wenn politische Ziel- und Strategieaussagen implementiert werden sollen. Diese Notwendigkeit folgt aus der Tatsache, dass Regelungen zur Daseinsvorsorge die situationsspezifischen Gegebenheiten (zeitlich, räumlich, sozial, kulturell) sowohl der raumgebundenen Ressourcen als auch der adressierten Benutzergruppen einbeziehen müssen, da sonst mit hoher Wahrscheinlichkeit Fehlleistungen und damit negative Effekte auftreten. Folglich kann es 
nur um Kontextsteuerung gehen, also um Rahmenaussagen, wobei die Konkretisierungen den dezentralen (regionalen) Einheiten überlassen bleiben sollten.

- Weiter wird auf ein Legitimitäts-Postulat hingewiesen: Strebe bei Programmen mit Wertimplikationen eine breite Akzeptanz der Adressaten an - eine Aufforderung, die bei pluralistischen Gesellschaften schwer herzustellen ist. Ohne Akzeptanz der Benutzergruppen wären Angebote zur Daseinsvorsorge bzw. erwartete Anpassungsleistungen beim Klimawandel nur autoritär, also mittels der hierarchischen regulativen Politik (Gebote, Verbote) durchzusetzen. Das aber ist demokratisch unbefriedigend; ein bloßer Rückgriff auf die Mechanismen der repräsentativen Demokratie erscheint nicht hinreichend. Hier trägt die sozialpsychologische Forschung wichtige Ergebnisse zu Attitüden, zu Vorurteilen, zur Konsens- und zur Lernbereitschaft bei (Rohmann \& Bierhoff 2010). Das spricht dafür, mehr experimentell, das heißt mit Lösungen auf Zeit, zu arbeiten (siehe drittes Brückenprinzip). Dezentrale, regionale Einheiten scheinen auch insoweit eine geeignete Handlungsebene darzustellen.

- Zusätzlich wird ein Verhandlungs-Postulat als Brückenprinzip vorgeschlagen. Dies ist eine entscheidende Bedingung für die Überwindung des „Free-rider“Prinzips, d.h. des egoistischen Missbrauchs von Gemeinschaftsgütern: Schaffe die Voraussetzungen für eine organisierte, verbindliche Kommunikation, damit kollektive Entscheidungen zustande kommen. Solche Voraussetzungen sind einmal eine langsam wachsende Vertrauensbasis und Reziprozität (,social capital“), zum anderen Anreizsysteme jenseits individueller Vorteile. Der Erfolg bei der Inszenierung regionaler Regimes hängt offenbar davon $a b$, wie gut sich unterschiedliche Akteursgruppen via Verhandlungsagenda (und einer effektiven Führung dabei) zusammenbringen lassen.

- Schließlich soll ein Nachhaltigkeits-Postulat als Brückenprinzip eingeführt werden. Es besagt, kurz gefasst: Steuere Entwicklungen sozialer, ökonomischer oder ökologischer Art und kontrolliere sie so in ihren Wirkungen, dass eine Generationengerechtigkeit erreicht wird, dass demnach Belastungen und Nachteile nicht überwiegend in die Zukunft verlagert werden. Dieses Postulat folgt zwar moralisch-rationalen Begründungen, doch diese entstammen nicht den philosophisch-anthropologischen Grundlagen; die Menschen neigen offenbar eher zu einer nicht-nachhaltigen Lebensweise. Die Begründung bedeutet daher, eine für notwendig erachtete Handlungsrichtung gegen vielfache lebenspraktische Realitäten Stück für Stück zu verwirklichen.

Mit den vier zuletzt genannten Brückenprinzipien wird deutlich, dass ihre Verwendung selbst einer weiteren Begründung bedarf: Warum gerade diese Postulate, warum keine anderen oder zusätzliche? Die interdisziplinäre Arbeitsgruppe Globaler Wandel - Regionale Entwicklung wählte nach Diskussion die hier empfohlenen Prinzipien aus und hält sie für besonders geeignet. Im Übrigen muss die Anwendung solcher Prinzipien für jede konkrete Situation eigenständig definiert werden.

Um eine mögliche Anwendung der Brückenprinzipien zur kritischen Bewertung von Handlungsoptionen zu veranschaulichen, sind im Folgenden konkrete Fragen im Sinne von Prüfkriterien formuliert, die hierbei hilfreich sein können. 
Tabelle 10. Kriterien zur Bewertung von Handlungsoptionen auf der Basis von Brückenprinzipien

\begin{tabular}{|c|c|}
\hline $\begin{array}{l}\text { Realisierbarkeits- } \\
\text { Postulat }\end{array}$ & $\begin{array}{l}\text { - Hat die Politik Handlungsoptionen eingeleitet, die mit dem Stand des wissen- } \\
\text { schaftlichen Wissens nicht vereinbar sind? } \\
\text { - Sollten diese Optionen daher kritisiert bzw. um alternative Optionen ergänzt } \\
\text { werden? } \\
\text { - Ergeben sich aus dem Stand des wissenschaftlichen Wissens Korridore bzw. } \\
\text { grundsätzliche Strategien für einzelne Handlungsoptionen? } \\
\text { - Wie sind diese normativen Ausrichtungen begründbar? }\end{array}$ \\
\hline $\begin{array}{l}\text { Ordnungs- } \\
\text { Postulat }\end{array}$ & $\begin{array}{l}\text { - Sind die potenziellen Folgen und Risiken einer gewählten Handlungsoption im } \\
\text { Hinblick auf ihre gestaltende Ordnungswirkung gut abzuschätzen? } \\
\text { - Inwieweit können die politisch oder ökonomisch vorgeschlagenen Problem- } \\
\text { lösungen (Handlungsoptionen) die Gefahr zusätzlicher Ungewissheit bzw. } \\
\text { chaotischer Situationen vermeiden oder abmildern? } \\
\text { - Falls nicht, lassen sich alternative Problemlösungen vorschlagen, die bei den zu } \\
\text { erwartenden Wirkungen besser abschneiden? }\end{array}$ \\
\hline $\begin{array}{l}\text { Experimentier- } \\
\text { Postulat }\end{array}$ & $\begin{array}{l}\text { - Gibt es einen juristischen Rahmen, um Lösungen auf Zeit zu „probieren“? } \\
\text { - Wie sollten Lösungsversuche bzw. Handlungsmodelle organisiert werden, } \\
\text { damit sie experimentelle Spielräume eröffnen? } \\
\text { - Wie lassen sich solche Erprobungen auswerten? }\end{array}$ \\
\hline $\begin{array}{l}\text { Kontext- } \\
\text { Postulat }\end{array}$ & $\begin{array}{l}\text { - Sind die situationsspezifischen Gegebenheiten sowohl der raumgebundenen } \\
\text { Ressourcen als auch der adressierten Benutzer einbezogen? } \\
\text { - Kann die Handlungsoption direkt auf der regionalen Ebene wirksam werden? } \\
\text { - Welchen Charakter können dann einheitliche bundesweite oder gar EU-weite } \\
\text { Vorgaben haben? }\end{array}$ \\
\hline $\begin{array}{l}\text { Legitimitäts- } \\
\text { Postulat }\end{array}$ & $\begin{array}{l}\text { - Ist für eine gewählte Handlungsoption eine möglichst breite Akzeptanz in der } \\
\text { Bevölkerung gegeben? } \\
\text { - Falls nein, mit welcher Vorgehensweise könnte, trotz pluraler Wertvorstellun- } \\
\text { gen, eine höhere Akzeptanz erreicht werden? } \\
\text { - Können Stakeholder dabei unterstützend tätig werden? } \\
\text { - Kann das nur bei einigen Gruppen gelingen? } \\
\text { - Welche Konsequenzen wären zu ziehen, falls diese Art von Legitimität verfehlt } \\
\text { würde? } \\
\text { - Müsste eine andere Handlungsoption gewählt werden? }\end{array}$ \\
\hline $\begin{array}{l}\text { Verhandlungs- } \\
\text { Postulat }\end{array}$ & $\begin{array}{l}\text { - Welche Kommunikationsformen werden von der öffentlichen Verwaltung bzw. } \\
\text { von den privatisierten Infrastrukturanbietern praktiziert? } \\
\text { - Sind sie überhaupt darauf angelegt, den Beteiligten ein breiteres Verständnis der } \\
\text { Problemsituation und der in Betracht kommenden Lösungswege zu ermögli- } \\
\text { chen? } \\
\text { - Wie könnte eine geeignete und verbindliche Kommunikation mit relevanten } \\
\text { Stakeholdern geführt werden? } \\
\text { - Und in welcher Weise können Benutzergruppen bzw. Mitglieder an Verhand- } \\
\text { lungen mitwirken? } \\
\text { - Liegen hierfür eine entsprechende Vertrauensbasis und ein geeignetes Anreiz- } \\
\text { system vor? }\end{array}$ \\
\hline $\begin{array}{l}\text { Nachhaltigkeits- } \\
\text { Postulat }\end{array}$ & $\begin{array}{l}\text { - Wie lässt sich bei den vorgeschlagenen Problemlösungen bzw. Handlungsop- } \\
\text { tionen sicherstellen, dass die belastenden Wirkungen nicht überwiegend in die } \\
\text { Zukunft verlagert werden? } \\
\text { - Wie können demnach nachhaltig wirksame Handlungsalternativen vorange- } \\
\text { bracht und bewertet werden? } \\
\text { - Wie lassen sie sich durchsetzen, etwa über das Legitimitäts- und das Verhand- } \\
\text { lungs-Postulat? } \\
\text { - Mithilfe welcher Stellschrauben kann wissenschaftliches Wissen zur Praxis } \\
\text { nachhaltigerer Problemlösungen beitragen? } \\
\text { - Wie ist ggf. zu entscheiden, falls nicht nachhaltige Wirkungen entsprechend der } \\
\text { aktuellen Interessenlage der Akteure und Bürger als unvermeidlich angesehen } \\
\text { werden? }\end{array}$ \\
\hline
\end{tabular}


Je nach konkreter Handlungssituation sind derartige Kriterien zu erweitern bzw. noch mehr zu operationalisieren. Das Aushandeln der so praktizierten Prinzipien ermöglicht immer auch, unter den Beteiligten - unter Beachtung unterschiedlicher Interessen - den größtmöglichen Grad an erreichbarer Konsensbildung festzustellen.

\section{Stärkung der interdisziplinären Forschung und des Transfers}

Wie am Beispiel der Fokusregion Berlin-Brandenburg dargestellt, münden der Umweltwandel, der Wandel von Landnutzung und Agrarmärkten sowie sozial- und raumstrukturelle Veränderungen in spezifische Herausforderungen. Aber auch auf globaler Ebene stellen Klimawandel, demographischer Wandel und globalisierte Warenströme die nachhaltige Nutzung der verfügbaren Ressourcen vor große Herausforderungen. Die Konflikte und Probleme bei der Verfügbarkeit, Qualität und Bewirtschaftung der Ressource Wasser wurden zu globalen Themen, die weit über bestehende administrative Grenzen hinausgehen und nur in internationaler Zusammenarbeit bewältigt werden können. Dennoch weisen die globalen Fragen jeweils sehr spezifische lokale und regionale Ausprägungen der Handlungsbedarfe auf. Darüber hinaus zeigen die Erfahrungen aus vielen Ländern, dass Fragen der Wasserbewirtschaftung nicht von einer Wissenschaftsdisziplin allein gelöst werden können. Vielmehr muss das Wissen unterschiedlicher Disziplinen integriert werden. Daher liegt es nahe, in einem nächsten Schritt die Fragestellungen und Ergebnisse der IAG Globaler Wandel - Regionale Entwicklung aufzugreifen und in einem internationalen Kontext zu erörtern. Aufbauend auf den Ergebnissen der IAG für die Fokusregion Berlin-Brandenburg wäre es aufschlussreich, die Thematik der Entwicklung von Wasserressourcen sowie deren Nutzung und politische Steuerung für ausgewählte europäische Regionen zu betrachten. In diesem Kontext wären die folgenden Aspekte zu behandeln:

- Überprüfung der Übertragbarkeit der für die Fokusregion Berlin-Brandenburg entwickelten Handlungsorientierungen (Kapitel I, II und III) für den Umgang mit den Folgen des globalen Wandels auf andere europäische Regionen. Hierzu bieten sich sowohl vermeintlich ähnliche als auch stark kontrastierende Regionen an.

- Nutzung der Handlungsorientierungen der IAG als Diskussionsgrundlage zur Identifizierung von politischen Handlungsmöglichkeiten auf der europäischen Ebene. Die Betrachtung der unterschiedlichen Problemlagen trägt dazu bei, zu erkennen, welche Herausforderung auf welcher politischen Handlungsebene angegangen werden sollte. In diesem Zusammenhang wäre es interessant, das Subsidiaritätsprinzip bzw. dessen Umsetzung weiterführend zu prüfen und konkrete Vorschläge für mögliche Instrumentarien abzuleiten.

- Die für die Fokusregion Berlin-Brandenburg identifizierten übergeordneten Herausforderungen sollten hinsichtlich ihrer internationalen Bedeutung überprüft und in ihrer jeweiligen regionalen Relevanz bewertet werden.

- Die genannten Brückenprinzipien könnten beispielhaft in verschiedenen Regionen zur Bewertung von Lösungsvorschlägen und zur Formulierung von Handlungsoptionen angewandt und auf ihre Allgemeingültigkeit hin überprüft werden. 
- Ein zusätzlicher Mehrwert aus der Internationalisierung der Thematik würde aus einer Verbesserung der Vernetzung von bestehenden Initiativen und Forschungsund Entwicklungs-Vorhaben auf Regional-, Landes-, Bundes- und EU-Ebene resultieren. Gerade auch mit Blick auf die Auswertung und Bereitstellung bestehender Daten wäre dies wünschenswert.

Die künftige Entwicklung und Nutzung von Wasserressourcen unter sich ändernden Rahmenbedingungen stellt, wie die vorangegangenen Ausführungen anschaulich belegt, eine Art „Brennglas“ dar, in dem sich die regionalen Auswirkungen des globalen Wandels beobachten lassen. Es wurde deutlich, dass sowohl eine stärkere interdisziplinäre Zusammenarbeit als auch die Fähigkeit, unter Bedingungen von Unsicherheit und Nichtwissen Entscheidungen treffen zu können, für die erfolgreiche Bewältigung der Herausforderungen unverzichtbar sind. Diese Erfordernisse sowie die zuvor genannten übergeordneten Herausforderungen und Brückenprinzipien legen nahe, das Thema der Wasserressourcen zukünftig stärker im Rahmen des Komplexes „Wissenschaft und Entscheidungsprozesse“ zu diskutieren. Dies schließt sowohl den Bereich der Generierung von neuem Wissen, dem die inhärenten Unsicherheiten inbegriffen sind, wie auch den Bereich verschiedener Entscheidungsprozesse, die von den spezifischen Akteurskonstellationen und der räumlichen und zeitlichen Wirkungsebene abhängen, ein. An diesem Anspruch müssen sich zukünftige wissenschaftliche wie politische Anstrengungen messen lassen.

\section{Literatur}

BMBF (2009): INKA BB. Innovationsnetzwerk Klimaanpassung Region Brandenburg Berlin. Fact Sheet (Onlinepublikation). http://www.klimzug.de/_media/INKA_druck.pdf (29.03.2011). Bonn: Bundesministerium für Bildung und Forschung (BMBF).

Calheiros, D., Seidl, A. \& Ferreira, C. (2000): Participatory research methods in environmental science: local and scientific knowledge of a limnological phenomenon in the Pantanal wetland of Brazil. Journal of Applied Ecology 37 (4), 684-696.

Ehleringer, J. R., Field, C. B. \& Roy, J. (Hrsg.) (1993): Scaling Physiological Processes: Leaf to Globe. San Diego: Academic Press.

Fritz, T. (2009): Peak Soil - Die globale Jagd nach Land. Berlin: FDCL-Verlag.

Haaren, C. v., Friese, K.-I., Hachmann, R., Meiforth, J., Neumann, A., Oppermann, B., Redslob, M., Tiedtke, S., Warren-Kretzschmar, B. \& Wolter, F.-E. (2005): Interaktiver Landschaftsplan Königslutter am Elm. Ergebnisse aus dem E+E-Vorhaben Interaktiver Landschaftsplan Königslutter am Elm (Vol. 24, Biologische Vielfalt). Bonn: Landwirtschaftsverlag.

Hallegatte, S. (2009): Strategies to adapt to an uncertain climate change. Global Environmental Change 19 (2), 240-247.

IGC (2011): Grain Market Report. GMR No. 409 (Onlinepublikation). http://www.igc.int/en/downloads/gmrsummary/gmrsumme.pdf (29.03. 2011). London: International Grains Council (IGC).

Joss, S. \& Durant, J. (Hrsg.) (1995): Public participation in science: the role of consensus conferences in Europe. London: Science Museum.

Keim, K.-D. K. (2009): Spacing-Konzepte und Brückenprinzipien zur Formulierung von Handlungsvorschlägen. Materialien der interdisziplinären Arbeitsgruppe Globaler Wandel - Regionale Entwicklung. Diskussionspapier 1. Berlin: Berlin-Brandenburgische Akademie der Wissenschaften.

Kirchner-Heßler, R., Konold, W. \& Gerber, A. (Hrsg.) (2007): Nachhaltige Landnutzung durch Kooperation von Wissenschaft und Praxis. Kulturlandschaft, Bd. 1. München: oekom. 
Medd, W. \& Marvin, S. (2005): From the politics of urgency to the governance of preparedness: a research agenda on urban vulnerability. Journal of Contingencies and Crisis Management 13 (2), 44-49.

Meehl, G. A., Stocker, T. F., Collins, W. D., Friedlingstein, P., Gaye, A. T., Gregory, J. M., Kitoh, A., Knutti, R., Murphy, J. M., Noda, A., Raper, S. C. B., Watterson, I. G., Weaver, A. J. \& Zhao, Z.-C. (2007): Global climate projections. In: S. Solomon, D. Qin, M. Manning, Z. Chen, M. Marquis, K. B. Averyt et al. (Hrsg.), Climate Change 2007: The Physical Science Basis. Contribution of Working Group I to the Fourth Assessment Report of the Intergovernmental Panel on Climate Change Cambridge, New York: Cambridge University Press.

Plieninger, T., Bens, O. \& Hüttl, R. F. (2009): Biomassenutzung aus Land- und Forstwirtschaft. In: H.-P. Beck, J. Buddenberg, E. Meller \& C. Salander (Hrsg.), Handbuch Energiemanagement. 28. Erg. Lfg. 05/09. Heidelberg: C.F. Müller Verlag, 1-24.

Plieninger, T., Thiel, A., Bens, O. \& Hüttl, R. F. (2008): Bioenergy clusters in Austria and Germany: From public goals to private action. In: T. Sikor (Hrsg.), Public and Private in Natural Resource Governance: A False Dichotomy? London: Earthscan, 149-166.

Renn, O., Schweizer, P.-J., Deyer, M. \& Klinke, A. (2007): Risiko. Über den gesellschaftlichen Umgang mit Unsicherheit. München: oekom.

Rittel, H. W. J. (1992): Planen, Entwerfen, Design. Ausgewählte Schriften zu Theorie und Methodik. Facility Management Nr. 5. Stuttgart: Kohlhammer.

Rohmann, E. \& Bierhoff, H.-W. (2010): Wahrnehmungen, Einstellungs- und Verhaltensänderungen in Bezug auf Global Change-Prozesse. Eine Analyse aus sozialpsychologischer Sicht. Materialien der interdisziplinären Arbeitsgruppe Globaler Wandel - Regionale Entwicklung. Diskussionspapier 6. Berlin: Berlin-Brandenburgische Akademie die Wissenschaften.

Rosswall, T., Woodmansee, R. G. \& Risser, P. G. (Hrsg.) (1988): Scales and Global Change: Spatial and Temporal Variability in Biospheric and Geospheric Processes. Scope 35. Chichester: John Wiley \& Sons.

Schelle, H., Ottmann, R. \& Pfeiffer, A. (2008): ProjektManager. Nürnberg: GPM Deutsche Gesellschaft für Projektmanagement.

Swyngedouw, E. (1997): Neither global nor local: "Glocalization" and the politics of scale. In: K. R. Cox (Hrsg.), Spaces of Globalization: Reasserting the Power of the Local. New York: Guilford Press, $137-166$.

Swyngedouw, E. (2010): Place, Nature and the Question of Scale: Interrogating the Production of Nature. Materialien der interdisziplinären Arbeitsgruppe Globaler Wandel - Regionale Entwicklung. Diskussionspapier 5. Berlin: Berlin-Brandenburgische Akademie der Wissenschaften.

Walkenhorst, O. \& Stock, M. (2009): Regionale Klimaszenarien für Deutschland. Eine Leseanleitung. Hannover: Akademie für Raumforschung und Landesplanung (ARL).

Wissen, M., Röttger, B. \& Heeg, S.(Hrsg.) (2008): Politics of Scale. Räume der Globalisierung und Perspektiven emanzipatorischer Politik. Münster: Westfälisches Dampfboot.

WWAP (2003): Water for People, Water for Life: The United Nations World Water Development Report. New York: World Water Assessment Programme WWAP (United Nations).

Zimmermann, L. (2007): Regionale Klima-Szenarien: kein „Spiel ohne Grenzen“! LWF aktuell $60,11-13$.

Sonja Germer $(\bowtie)$

Berlin-Brandenburgische Akademie der Wissenschaften

germer@bbaw.de

Oliver Bens

Helmholtz-Zentrum Potsdam - Deutsches GeoForschungsZentrum (GFZ) 
Rolf Emmermann

Helmholtz-Zentrum Potsdam - Deutsches GeoForschungsZentrum (GFZ)

Reinhard F. Hüttl

Helmholtz-Zentrum Potsdam - Deutsches GeoForschungsZentrum (GFZ)

Karl-Dieter Keim

Leibniz-Institut für Regionalentwicklung und Strukturplanung e.V. (IRS), Erkner, und Brandenburgische Technische Universität Cottbus

Matthias Naumann

Leibniz-Institut für Regionalentwicklung und Strukturplanung e.V. (IRS), Erkner 